\title{
Parasitismo por Amblyomma ovale e Amblyomma fuscum (Acari: Ixodidae) em Dasypus novemcinctus (Xenarthra: Dasypodidae) no Brasil
}

\author{
Parasitism by Amblyomma ovale and Amblyomma fuscum (Acari: Ixodidae) \\ on Dasypus novemcinctus (Xenarthra: Dasypodidae) in Brazil
}

\author{
Sâmara Nunes Gomes ${ }^{1 *}$, Tatiana Cheuiche Pesenti ${ }^{1}$, Gertrud Müller ${ }^{1}$ \\ |||||||||||||||||||||||||||||||||||||||||||||||||||||||||||||||||||||||||||||||||||||||||||||||||||||||||||||||||||||||||||||||||||||||||||||||||||||||||||||||||||||||||||||||||||||||||||||||||||||||||||||||||||||||||||||||||||||||||||||||||||
}

RESUMO: Carrapatos são ectoparasitos hematófagos importantes para a saúde pública e animal por transmitirem agentes infecciosos durante a hematofagia, bem como causarem injúrias a seus hospedeiros. A ocorrência dos carrapatos Amblyomma ovale e Amblyomma fuscum é registrada, pela primeira vez, para o Brasil, parasitando tatu-galinha (Dasypus novemcinctus).

PALAVRAS-CHAVE: Ixodidae, Amblyomma ovale, Amblyomma fuscum, Dasypus novemcinctus, tatu-galinha, Brasil.

\begin{abstract}
Ticks are important bloodsucking ectoparasites for public and animal health for transmitting infectious agents during blood feeding and causing injuries to their hosts. The authors report the occurrence of Amblyomma ovale and Amblyomma fuscum for the first time in Brazil, parasitizing the nine-banded armadillo (Dasypus novemcinctus).
\end{abstract}

KEYWORDS: Ixodidae, Amblyomma ovale, Amblyomma fuscum, Dasypus novemcinctus, nine-banded armadillo, Brazil. 
Existem cerca de 870 espécies de carrapatos descritas no mundo, todas agrupadas na subordem Ixodida, sendo que somente a família Ixodidae compreende aproximadamente 680 espécies, com os gêneros Amblyomma, Anomalohimalaya, Bothriocroton, Cosmiomma, Dermacentor, Haemaphysalis, Hyalomma, Ixodes, Margaropus, Nosomma, Rhipicentor e Rhipicephalus (BARRosBATTESTI et al., 2006). A região neotropical está representada por 117 espécies, incluídas em cinco gêneros: Amblyomma, Dermacentor, Haemaphysalis, Ixodes e Rhipicephalus (SERRAFreire; Mello, 2006). No Brasil, são conhecidas 56 espécies de carrapatos, das quais 33 são do gênero Amblyomma (Barros-Battesti; Knissak, 1999). A variedade de hospedeiros deste gênero é grande, compreendendo a maioria das ordens de mamíferos (Barros-Battesti et al., 2006). Relativamente poucos carrapatos foram documentados para Dasypus novemcinctus, todos pertencentes à família Ixodidae, e gênero Amblyomma. Guglielmone; Nava (2006) reportaram A.parvum em $D$. novemcinctus na Argentina; adicionalmente, Nava et al. (2007) listaram A. auricularium na mesma espécie no Paraguai. No Brasil, Bотецho et al. (1989) relataram a presença de $A$. cajennense e $A$. pseudoconcolor também em $D$. novemcinctus na Serra da Canastra, Minas Gerais, assim como Szabó et al. (2007) relataram, na mesma espécie, A. auricularium em Goiás.

BRUM et al. (2003) reportaram o parasitismo de Amblyomma fuscum em Dasypus septemcinctus no município de Pelotas, Rio Grande do Sul. Medri et al. (2010) identificaram A. cajennense, A. parvum e Amblyomma sp. em Euphractus sexcinctus no Pantanal Matogrossense. Na Argentina, MAuri; Navone (1993) registraram a presença de $A$. auriculare nas seguintes espécies de Xenarthra: Chaetophractus villosus, C. vellerosus, Tolypeutes matacus e Zaedyus pichiy; e A. tigrinum em C. vellerosus.
O objetivo deste trabalho foi identificar os carrapatos encontrados em tatu-galinha no Rio Grande do Sul, Brasil.

Foi recolhido um espécime macho de Dasypus novemcinctus na BR 116, no município de Camaquá, morto por atropelamento. O mamífero foi transportado ao Laboratório de Parasitologia de Animais Silvestres da Universidade Federal de Pelotas, onde teve seu corpo examinado para estudos parasitológicos. Os carrapatos encontrados foram retirados, cuidadosamente, do corpo do animal por torçáo do seu eixo longitudinal, colocados em álcool $70^{\circ} \mathrm{GL}$ e identificados de acordo com Barros-Battesti et al. (2006).

Duas espécies de Amblyomma foram identificadas parasitando Dasypus novemcinctus: A. ovale (Kock, 1844) (Fig. 1A-B) e A. fuscum (Neumann, 1899) (Fig. 2A-B), sendo que $A$. ovale foi mais abundante (11 fêmeas e 15 machos) do que $A$. fuscum (uma fêmea e quatro machos).

Amblyomma ovale é, no Brasil, uma espécie de hábitos nitidamente silvestres, raramente encontrada próxima às cidades, podendo ser localizada parasitando cáes, utilizados para caçar (ARAGÃo; Fonseca, 1961). Este fato justifica a maior quantidade desta espécie parasitando $D$. novemcinctus, que, juntamente a $D$. hybridus (Tatu-mulita), representam as espécies cinegéticas com maiores registros de caça por perseguição de cáes (Peters et al. 2011). A. ovale apresenta dois espinhos agudos e longos na Coxa I, onde o espinho externo é encurvado na extremidade (Fig. 1A). O escudo é castanho com faixas cor de cobre (Fig. 1B).

De acordo com Guimaráes et al. (2001), A. fuscum tem sido descrito parasitando preferencialmente répteis, no entanto há relatos em anfíbios (Sinkoc; Brum, 1997), e em outros mamíferos como Cerdocyon thous, Canis familiaris e Dasypus septemcinctus (BRUM et al., 2003; BARRos-BATTESTI et al., 2005). Dorsalmente, apresenta um escudo de coloraçáo marrom amarelado, com
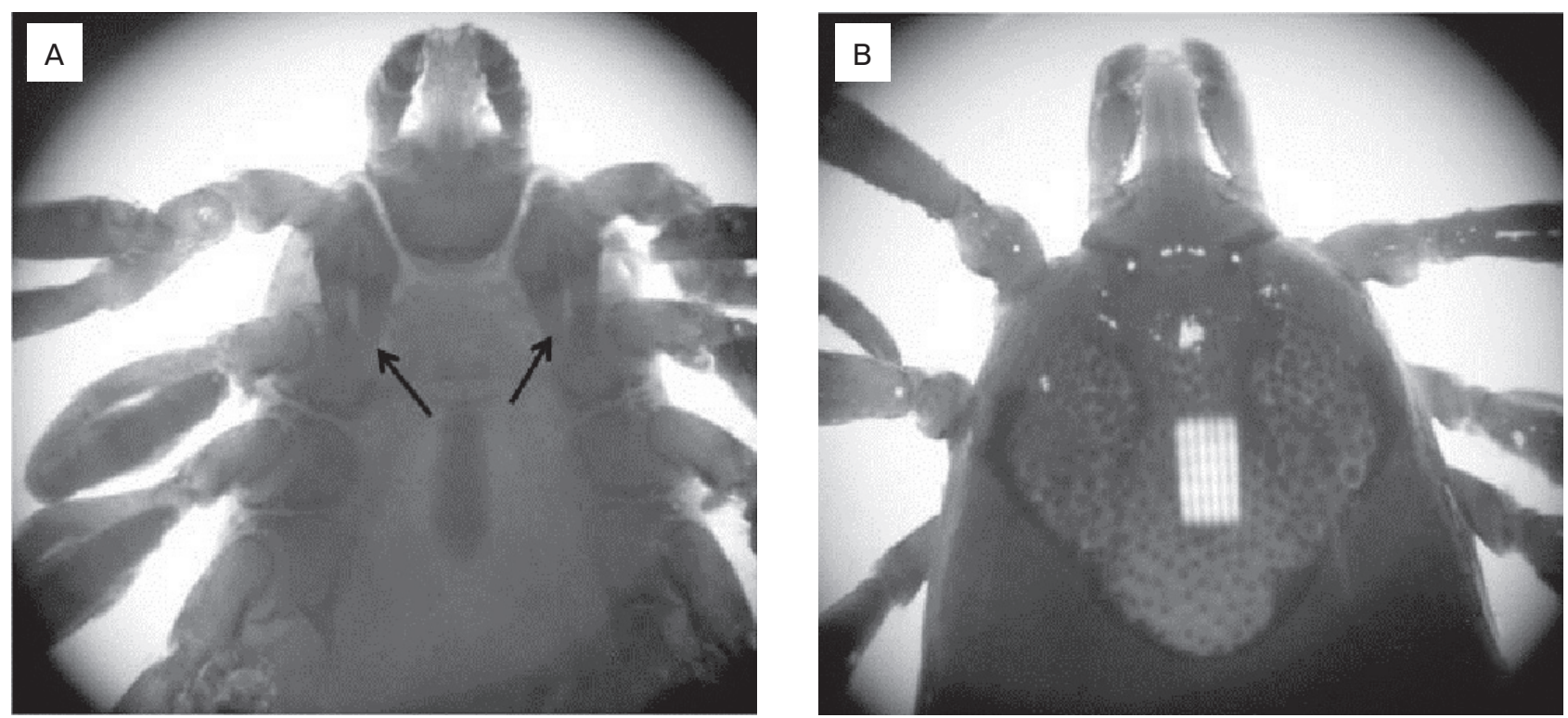

Figura 1. Amblyomma ovale. (A) Espinhos longos na Coxa I. (B) Fêmea dorsal. 

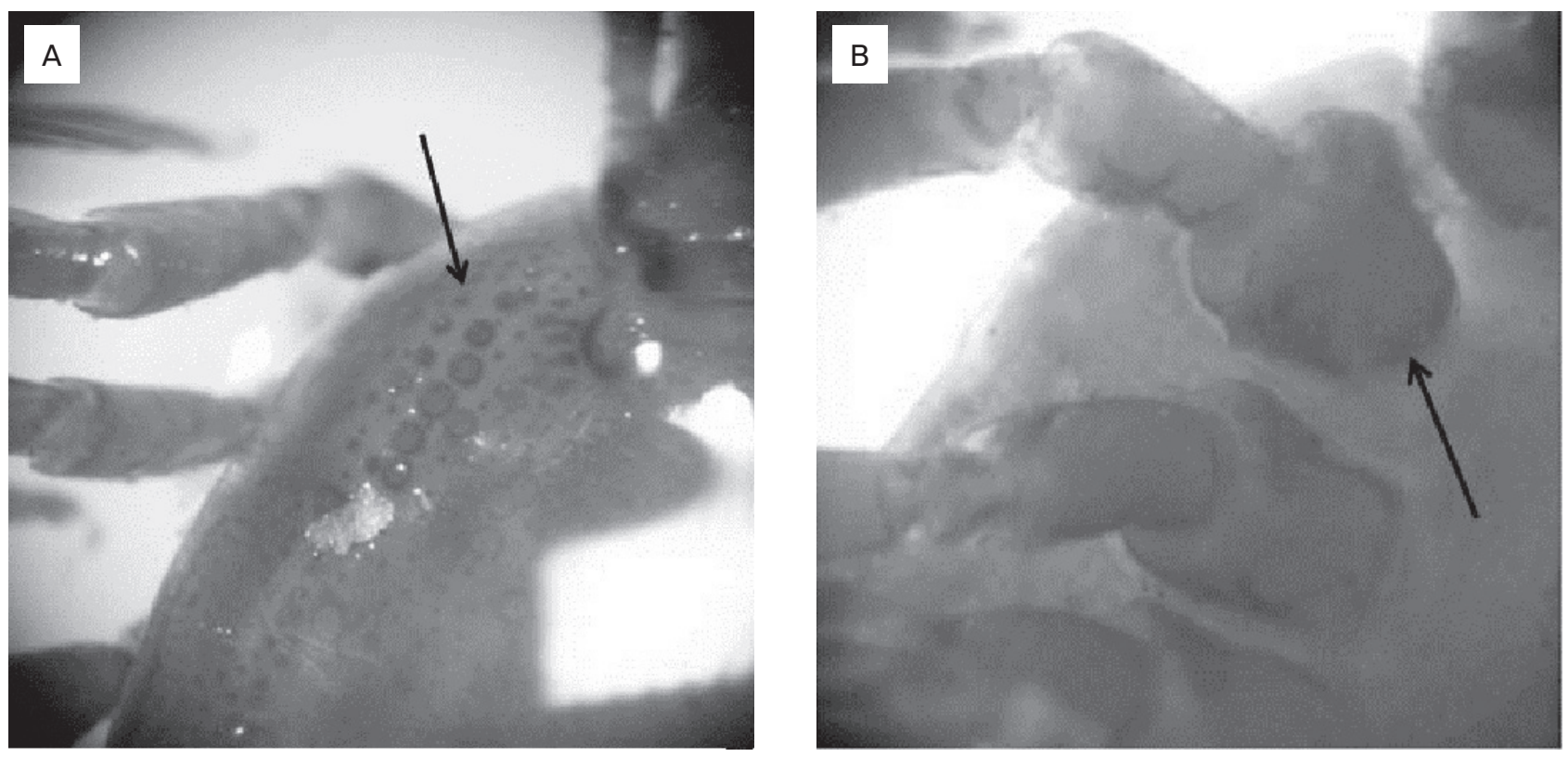

Figura 2. Amblyomma fuscum. (A) Pontuações laterais no escudo. (B) Espinhos curtos na Coxa I.

bordos acobreados. As pontuaçóes são poucas, profundas e concentradas nas porçôes laterais (Fig. 2A). Ventralmente, há dois espinhos curtos e largos na Coxa I (Fig. 2B).

Carrapatos da família Ixodidae possuem altas taxas de reprodução e podem ser transportados por longas distâncias por meio de seus hospedeiros. Desta forma, a fauna silvestre participa decisivamente nessa atividade, promovendo a disseminação e adaptaçáo dos carrapatos a diversos tipos de ambientes. (RoHR, 1909; Moreira, Magalhaes, 1935; Cloudsley-Thompson, 1980).

Como visto neste estudo, várias espécies de carrapatos podem coabitar no mesmo hospedeiro. Cáes que entram na mata ou que compartilham seu ambiente com a fauna silvestre (SZABó et al., 2001) podem apresentar infestações mistas. Da mesma forma, pequenos mamíferos silvestres que vivem próximos aos domicílios também podem apresentar dupla infestação. BARros-Battesti et al. (2000) verificaram a presença de $A$. cajennense I Ixodes loricatus no gambá, assim como ARzua et al. (2003) observaram dupla infestação em aves passeriformes por A. aureolatum e Ixodes auritulus. Por meio deste estudo, relata-se o parasitismo de Amblyomma ovale e Amblyomma fuscum, pela primeira vez, no Brasil, em tatu-galinha, oriundos do estado do Rio Grande do Sul.

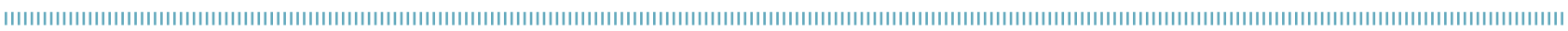

\section{REFERÊNCIAS}

ARAGÃO, H.B.; FONSECA, F. Notas de ixodologia: IX. O complexo ovale do gênero Amblyomma. Memórias do Instituo Oswaldo Cruz, v.59, n.2, p.131-148, 1961.

ARZUA, M.; SILVA, M.A.N.; FAMADAS, K.M.; BEATI, L.; BARROSBATTESTI, D.M. Amblyomma aureolatum and Ixodes auritulus (Acari: Ixodidae) on birds in southern Brazil, with notes on their ecology. Experimental and Applied Acarology, v.31, n.3-4, p.283296, 2003.

BARROS-BATTESTI, D.M.; KNYSAK, I. Catalogue of the Brazilian Ixodes (Acari: Ixodidae) material in the mite collection of Instituto Butantan, São Paulo, Brazil. Papéis Avulsos de Zoologia, São Paulo, v.41, n.3, p.41-57, 1999.

BARROS-BATTESTI, D.M.; YOSHINARI, N.H.; BONOLDI, V.L.N.; GOMES, A.C. Parasitism by Ixodes didelphidis and I. Loricatus (Acari: Ixodidae) on small wild mammals from an Atlantic Forest in the State of São Paulo, Brazil. Journal of Medical Entomology, v.37, n.6, p.820-827, 2000.

BARROS-BATTESTI, D.M.; ONOFRIO,V.C.; LABRUNA, M.B.; MARTINS, J.R.; GUGLIELMONE, A.A. Redescription of Amblyomma fuscum Neumann, 1907 (Acari: Ixodidae), a rare South America tick confirmed in Brazil. Systematic Parasitology, v.61, n.2, p.8592, 2005.

BARROS-BATTESTI, D.M.; ARZUA, M.; GERVÁSIO, H.B. Carrapatos de importância médico-veterinária da região neotropical, um guia ilustrado para identificação de espécies. São Paulo: ICTTD, 2006. $223 \mathrm{p}$.

BOTELHO, J.R.; LINARDI, P.M.; ENCARNAÇÃO, C.D. Interrelação entre Acari Ixodidae e hospedeiros edentata da Serra da Canastra, Minas Gerais, Brasil. Memórias do Instituto Oswaldo Cruz, Rio de Janeiro, v.84, n.1, p.61-64, 1989. 
BRUM, J.G.W.; VALENTE, A.L.S.; ALBANO, A.P.; COIMBRA, M.A.C.; GREQUE, G.G. Ixodidae de mamíferos silvestres atendidos no Núcleo de Reabilitação da Fauna Silvestre, UFPEL. Arquivos do Instituto Biológico, v.70, n.2, p.211-212, 2003.

CLOUDSLEY-THOMPSON, J.I. Microecologia. São Paulo: EPU, 1980. 58p.

GUGLIELMONE A.A.; NAVA S. Las garrapatas argentinas del género Amblyomma (Acari: Ixodidae): distribución y hospedadores. Revista de Investigaciones Agropecuarias, v.35, n.3, p.133-153, 2006.

GUIMARÃES, J.H.; TUCCI, E.D.; BARROS-BATTESTI, D.M. Ectoparasitos de importância veterinária. São Paulo: Plêiade, 2001. 213p.

MAURI, R.; NAVONE, G.T. Common ectoparasites (Siphonaptera and Acari) of Dasypodidae (Mammalia: Xenarthra) in Argentina. Revista de la Sociedad Entomológica Argentina, v.52, n.1-4, p.121-122, 1993.

MEDRI, I.M.; MARTINS, J.R.; DOYLE, L.R.; MOURÃO, G.; MARINHOFILHO, J. Ticks (Acari: Ixodidae) from yellow armadillo, Euphractus sexcinctus (Cingulata: Dasypodidae), in Brazil's Pantanal wetlands. Neotropical Entomology, v.39, n.5, p.823-825, 2010.

MOREIRA, J.A.; MAGALHÃES, O. Thypho exanthematico em Minas Gerais. Brasil Médico, v. 44, p. 465-470, 1935.

NAVA, S., LARESCHI, M., REBOLLO, C., BENITTEZ, U.C., BEATI, L., ROBBINS, R.G., DURDEN, L.A., MANGOLD, A.J., GUGLIELMONE, A.A.
The Ticks (Acari: Ixodida: Argasidae, Ixodidae) of Paraguay. Annals of Tropical Medicine and Parasitology, v.101, n.3, p.255270, 2007.

PETERS, F.B.; ROTH, P.R.O.; PEREIRA, M.S.; PISKE, A.D.; CHRISTOFF, A.U.. Aspectos da caça e perseguição aplicada à mastofauna na área de proteção ambiental do Ibirapultã, Rio Grande do Sul, Brasil. Biodiversidade Pampeana, Uruguaiana, v.9, n. 1, p.16-19, 2011.

ROHR, C.J. Estudo sobre Ixodidas no Brasil. Rio de Janeiro: Gomes \& Irmão, 1909. 220p.

SERRA-FREIRE, N.M.; MELLO, R.P. Entomologia @ acarologia na medicina veterinária. Rio de Janeiro: L.F. Livros, 2006. 200p.

SINKOC, A.L.; BRUM, J.G.W. Ocorrência de Amblyomma fuscum Neumann, 1899 e Amblyomma humerale Koch, 1844 (Acari: Ixodidae) em Bufo arenalis no estado de São Paulo, Brasil. Ciência Rural, v.27, n.2, p.339-340, 1997.

SZABÓ, M.P.J; CUNHA, T.M.; PINTER, A.; VICENTINI, F. Ticks (Acari: Ixodidae) associated with domestic dogs in Franca region, São Paulo, Brazil. Experimental and Applied Acarology, v.25, n.1011, p.909-916, 2001.

SZABÓ, M.P.J.; OLEGÁRIO, M.M.; SANTOS, A.L. Tick fauna from two locations in the Brazilian savannah. Experimental and Applied Acarology, v.43, n.1, p.73-84, 2007. 